\title{
Kinetics of unusual photoinduced currents in the solid state of some ferrocene derivatives
}

\author{
Aloke Kumar Chakraborty and Biswanath Mallik* \\ Department of Spectroscopy, Indian Association for the Cultivation of Science, \\ 2A \& 2B, Raja S. C. Mullick Road, Jadavpur, Kolkata-700 032, India \\ E-mail : spbm@mahendra.iacs.res.in
}

Received 18 September 2008, accepted 12 January 2009

\begin{abstract}
Kinetics of change in photoinduced currents (during illumination and after switching off light source) in the solid state of some ferrocene derivatives, namely, ferrocenecarboxylic acid, acetylferrocene, ferrocenecarbaldehyde, hydroxymethyl ferrocene have been studied in dry nitrogen gas atmosphere. Unusual/ anomalous photocurrent versus time profiles were observed in some ferrocene derivatives at certain cell temperatures. The kinetics of current changes under photoexcitation and after switching off the light source have been observed to be complicated in nature. Temperature-dependent behavior of the studied kinetics indicates that charge carrier trapping/ detrapping and recombination processes are significantly temperature dependent. These processes have an important role in causing the temperature dependent unusual/ anomalous photocurrent versus time profiles in the ferrocene derivatives studied.
\end{abstract}

Keywords : Organic semiconductor; photoconductivity; recombination and trapping; phase transition.

PACS Nos. : 72.80.Le; 72.00.-i; 72.40.+w; 82.20.-w; 73.50.Gr

\section{Introduction}

In the advancement of experimental and theoretical aspects of physics and chemistry including photophysical properties, the discovery of metallocenes along with their derivatives have shown an important role [1-3]. The photophysical properties lead to the understanding in photoinduced changes that occur due to photoexcitation [4,5]. Some significant effects due to the attachment of functional groups to the ferrocene unit have been reported in case of electroluminescence, photoresponse, UV-Vis spectra, luminescence, redox potentials, electrical conductivity, fluorescence spectra, the incident of photon-to-current conversion efficiency (IPCE) [6-8].

* Corresponding Author 
Previous studies in our laboratory have shown that different functional groups attached to ferrocene unit have significant effects on various physical phenomena like adsorptioninduced changes in electrical conductivity (semiconductivity) [9], photoinduced desorption of ethanol vapour from the solid surface of some ferrocene derivatives [10], temperaturedependence of the characteristic of photoconducting systems $(\gamma)$ [11], photoinduced-charge transfer between ferrocene derivatives and chloroform molecules confined in poly (methyl methacrylate) (PMMA) thin films [12] etc. In addition, in our laboratory anomalous behavior in photoconductivity of ferrocene was observed [13]. To study the photoconductivity behavior of ferrocene derivatives, changes in current with time were studied at different values of fixed temperature of sample cells under photoexcitation and also after switching off the light source in some pure ferrocene derivatives using sandwich-type cell configuration. In the course of such studies new types of current versus time profiles, depending on cell temperature, were obtained. The charge carrier trapping / detrapping and recombination processes are known to influence the kinetics of rise and decay of photocurrent of organic as well as organometallic photoconductors [14]. Consequently, the studies of kinetics of change in current during light exposure and after switching off the light source in the case of some ferrocene derivatives were performed at different sample cell temperatures. In this communication the results of such studies have been presented.

\section{Experimental}

High purity microcrystalline sample (in powder form) of ferrocene $(\mathrm{FcH})$, ferrocenecarboxylic acid $[\mathrm{Fc}(\mathrm{COOH})]$, ferrocenecarbaldehyde $[\mathrm{Fc}(\mathrm{CHO})]$, hydroxymethyl ferrocene $\left[\mathrm{Fc}\left(\mathrm{CH}_{2} \mathrm{OH}\right)\right]$, acetylferrocene $\left[\mathrm{Fc}\left(\mathrm{COCH}_{3}\right)\right]$ were used after further purification by repeated crystallization. The experiments were performed by usual sandwich cell technique [9-11]. To prepare a sample cell, pure and dry powdery material $(\sim 10 \mathrm{mg})$ was placed between a conducting glass (indium tin oxide (ITO) coated) and a stainless steel electrode. Teflon spacers, 2 mil $(0.00508 \mathrm{~cm})$ thick, maintained the separation between the electrodes. To maintain the sandwich cell, two spring clips were attached at a moderate pressure (about 0.035 $\mathrm{MPa}$ ) at the two sides of the electrodes. The sample cells were prepared in air and in safe light illumination. The sample cells were kept inside a brass chamber, fashioned with Teflon and having a quartz window at the top for photoconductivity studies. Before performing any measurement, several heating and cooling treatments were applied to the sample cell; initially in vacuum by operating pump and finally in the controlled flow of dry nitrogen gas circulated through the conductivity chamber. This process was carried out to ensure the complete desorption of any preadsorbed (if adsorbed during the preparation of the sample cell) water vapor, oxygen or other gas/vapor from the sample cell. The heat treatments were applied (by electrical heating arrangement operated from outside) up to a temperature well below the melting point and phase transition temperature (if any) of the materials under study. Temperature of the sample cells was controlled by a proportional temperature controller (model : RTE 110, Neslab Inst. Inc., NH, USA) and also cooling treatments were carried out by it. A dc bias voltage of $27 \mathrm{~V}$ from a dry battery was applied across the sample cell. A programmable electrometer (model 617, Keithley Inst. 
Inc., Cleveland, $\mathrm{OH}, \mathrm{USA}$ ) was used for the current measurements. Experiments were performed with $27 \mathrm{~V}$ bias voltage as with such a value, appreciable current (Ohmic range) in nitrogen gas environment near room temperature was obtained. The temperature was measured by using a copper-constantan thermocouple attached at the top of the metal electrode and a microprocessor-controlled digital millivoltmeter. In this work, the conductivity cell consisted of two junctions of stainless steel electrode/ metallocenes and conducting glass / metallocenes. To check whether the junctions were rectifying or Ohmic, current measurements in a sandwich cell in case of each material were performed with forward as well as with reverse bias. The values of the current measured with both forward and reverse bias were almost equal which indicated that the junctions were Ohmic.

Mercury lamp of $125 \mathrm{~W}$ was used as polychromatic source of light for this work. The approximate photon flux reaching the sample varied from $3.33 \times 10^{12}$ to $3.18 \times 10^{12}$ per $\mathrm{cm}^{2}$ with increasing wavelength in the visible region of the spectrum. A monochromator (Type H2O UV, Instruments S.A., Jobin-Yvon, France) was placed between the lamp and the quartz window of the chamber for wavelength-dependence studies but no appreciable change in current was noticed. Vacuum deposited thin film of ferrocene sublimes at the studied range of temperature of this work. For comparative studies powdered (qualitatively same grain size) sandwich cells of the ferrocene derivatives were used. The light exposure was allowed for $70 \mathrm{sec}$ and the corresponding currents during illumination and after switching off the light source were recorded as a function of time by using data storage mode of the electrometer. The experiment was performed under controlled dry nitrogen atmosphere.

\section{Results and discussion}

The steady state electrical (dark) conductivity, in a sandwich cell of compressed powder of organometallic materials can be expressed as $[13,15]$ as

$$
\sigma(T)=\sigma_{0} \exp \left(-\frac{E_{d}}{2 k T}\right)
$$

where, $\sigma_{0}$ is the preexponential factor, $E_{d}$ is the activation energy for dark conduction, $k$ is the Boltzmann constant and $T$ is the absolute temperature of the sample cell. The preexponential factor of eq. 1 can be expressed $[15,16]$ as

$$
\sigma_{0}=q N \mu
$$

with $N=N_{A} \rho Z / M$ based on the conventional solid state theory, where $q$ is the charge on the carrier, $\mu$ is the mobility, $N_{A}$ is Avogadro's number, $M$ is the molecular weight of the solid material, is the density, and $z$ is the number of charge carriers available for excitation per molecule. In the present experiment, the is proportional to the steady state dark current $\left(I_{d}\right)$, which has been measured in the sandwich cells of compressed powders of organometallic materials under study and $I_{d}$ can be represented by 


$$
I_{d}=I_{0} \exp \left(-\frac{E_{d}}{2 k T}\right)
$$

where, $I_{0}$ is the preexponential factor and other terms have usual meaning as stated above. Usually on irradiation of light, photocurrent $\left(I_{p h}\right)$ increases with time due to the increase of excess charge carrier density generated upon photoexcitation and ultimately attains a saturation value/ steady state value [17]. After turning off the light source, photocurrent decays to the dark current value $\left(I_{d}\right)$ as observed before photoexcitation [17]. The temperature dependence of steady state photocurrent $I_{p h}$ (similar to $I_{d}$ ) in case of organic/ organometallic materials can also be expressed by the expression

$$
I_{p h}=I_{0}^{\prime} \exp \left(-\frac{E_{p h}}{2 k T}\right)
$$

where $I_{0}^{\prime}$ is the preexponential factor and $E_{p h}$ is the activation energy for photoconduction. Similar to the dark conductivity, photoconductivity is proportional to photocurrent in the present experimental arrangement. Photocurrent is defined by the difference between the total current measured under illumination and the dark current. While we studied the photoconductivity behavior of some ferrocene derivatives by monitoring the changes in current with time, at different values of fixed temperature of sample cells, under photoexcitation and also after switching off the light source, unusual changes in photocurrent with time were noticed in some cases. The results are discussed below.

For the polycrystalline powder sandwich type cell of $\mathrm{Fc}\left(\mathrm{COCH}_{3}\right)$ at a temperature of $292.90 \mathrm{~K}$, the current versus time profile i.e., values of the current (dark current before photoexcitation, $I_{p h}$ during the $70 \mathrm{sec}$ of light exposure, and measured current after switching off the light source) as a function of time is shown in Figure 1(a) (curve A, left scale). With the initiation of the light exposure, the value of $I_{p h}$ started to increase rapidly and attained a maximum value with time. Current values started decreasing abruptly when the light was switched off and with the passage of time the current profile attained almost a steady value with some fluctuations. In case of the same material, similar current profile with time was noticed at a higher temperature of $306.52 \mathrm{~K}$ with comparatively more fluctuations after switching off the light source (Figure 1(a), curve B, right scale). Within almost the same temperature range the current versus time profile was entirely different (the current profile decreased suddenly with the initiation of light exposure indicating that the value of $I_{p h}$ is much lower than the original dark current) for $\mathrm{Fc}\left(\mathrm{CH}_{2} \mathrm{OH}\right)$ as shown in Figure 1(b), curve A, at a temperature of $303.21 \mathrm{~K}$. From curve A of Figure 1(b), it is evident that the fluctuations in current values after switching off the light were also present. At a higher temperature of $318.94 \mathrm{~K}, \mathrm{Fc}\left(\mathrm{CH}_{2} \mathrm{OH}\right)$ showed similar current versus time profile (Figure 1(b), curve $\mathrm{B}$ ) with the exception of slightly increasing current profile after a sudden decrease in the presence of light exposure. Thus, the current versus time profiles 
depend significantly on both the nature of the substituted group in ferrocene unit as well as the sample temperature.

The kinetics of change in currents during illumination and after switching off light has been analyzed in usual conventional way $[18,19]$. The reaction mechanism remains the primary goal of most kinetics research, and considerable progress has been made in the ability to predict rates of reaction. Kinetics is concerned fundamentally with the details of the progress whereby a system gets from one state to another and with the time required for the transition. Equilibrium can also be treated in principle on the basis of kinetics as that situation in which the rates of forward and reverse reactions are equal. Theoretical methods for predicting reaction rates are based on the conclusion drawn by Arrhenius : the most commonly observed relationship between rate of reaction and temperature implies to energy barrier to reaction.

The linearity of logarithm of $I_{p h}$ versus time has been reported [13] to be linear at all the sample cell temperatures studied. Such linearity indicates that the change in $I_{p h}$ obeys first order kinetics $[18,19]$. The plots of logarithm of $I_{p h} v s$ time in case of FcH derivatives studied in the present experiment were not linear at any cell temperature of study. The observed results indicate that the time dependent changes in $I_{p h}$ in the ferrocene derivatives under study do not obey first order kinetics. Rather, in the present materials, the plots of logarithm of $I_{p h}$ vs logarithm of time have been observed to be linear i.e., a power-law dependence of photocurrent on time was indicated. Power-law distributions occur in many scientific phenomena and have significant consequences for our understanding of natural as well as man-made phenomena $[20,21]$. The plots of logarithm of $I_{p h} v s$. logarithm
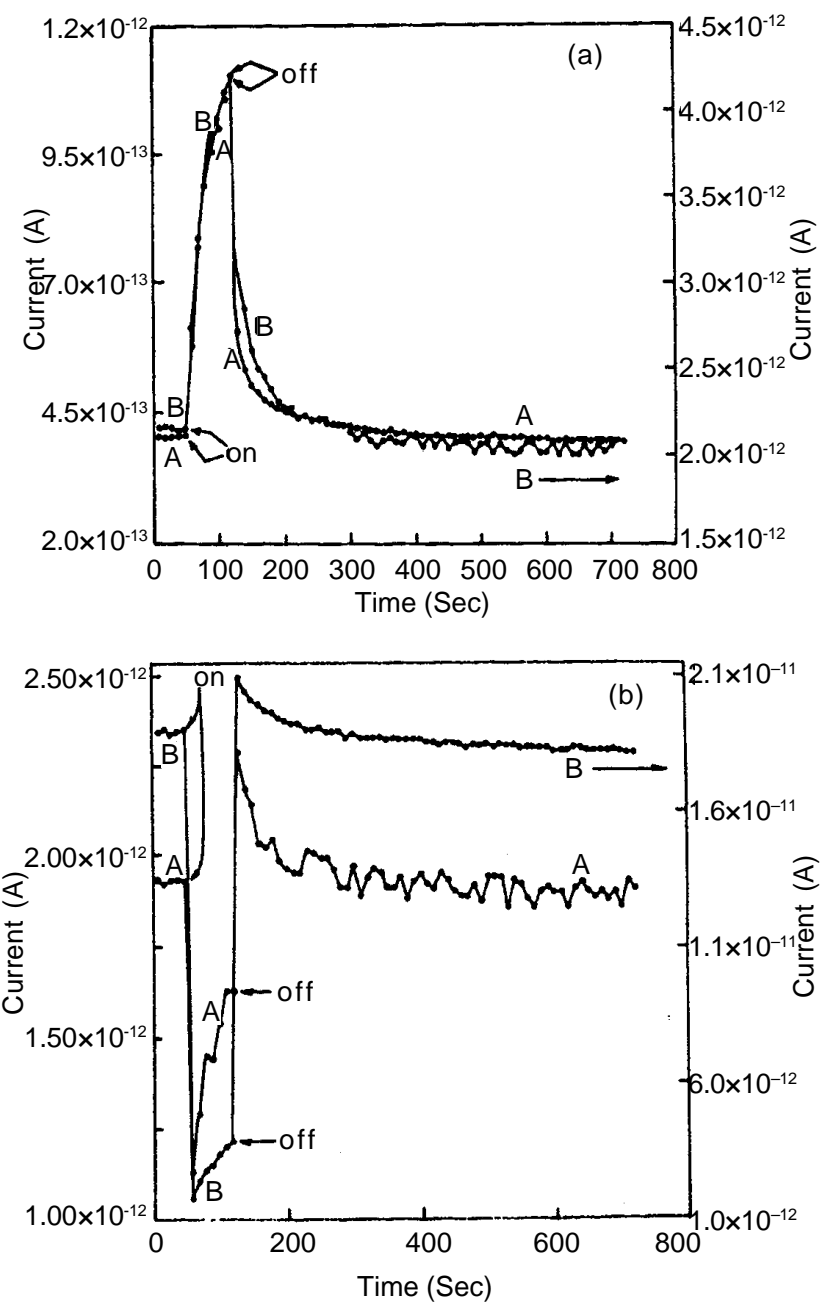

Figure 1. Change in current (using mercury lamp of $125 \mathrm{~W}$ ) with time at different temperatures in powder cells of ("on" and "off" states of light source are indicated by "on" and "off" respectively) : (a) acetylferrocene - Curve A, $292.90 \mathrm{~K}$ (left scale); Curve B, $306.52 \mathrm{~K}$ (right scale); (b) hydroxymethylferrocene- Curve A, 303.21 K (left scale); Curve B, $318.94 \mathrm{~K}$ (right scale). 
of time are shown in Figure 2(a) to (d) for $\mathrm{Fc}(\mathrm{COOH}), \mathrm{Fc}\left(\mathrm{COCH}_{3}\right), \mathrm{Fc}(\mathrm{CHO})$, and $\mathrm{Fc}\left(\mathrm{CH}_{2} \mathrm{OH}\right)$, respectively. It is noticeable from Figure 2(a) and Figure 2(d) that in case of $\mathrm{Fc}(\mathrm{COOH})$ and $\mathrm{Fc}\left(\mathrm{CH}_{2} \mathrm{OH}\right)$ the data points are highly scattered at some temperatures. For all the $\mathrm{FcH}$ derivatives under study, the order of the kinetics of change in $I_{p h}$ seems to be complicated in nature.
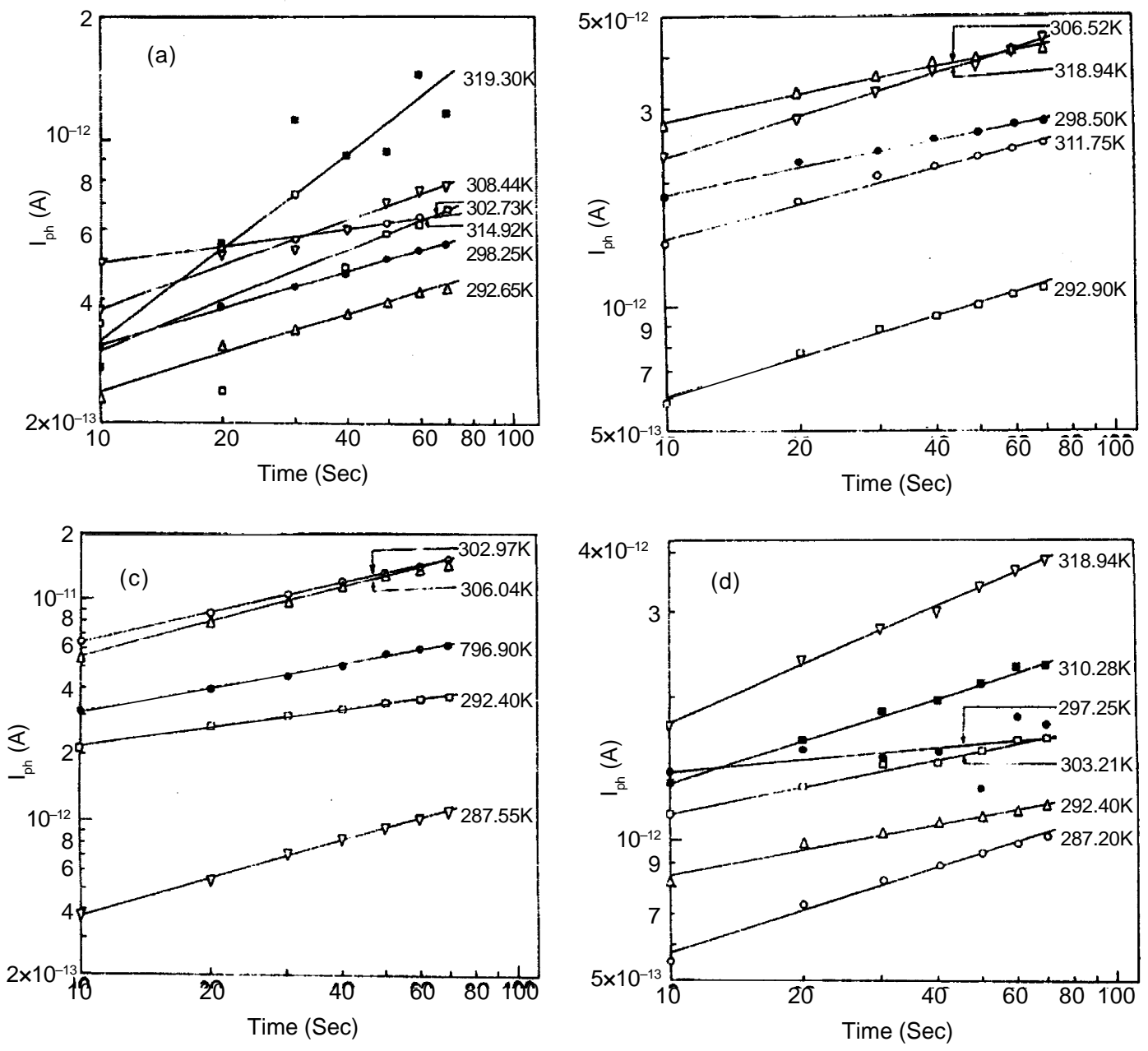

Figure 2. Plots of logarithm of $I_{p h}$ vs. logarithm of time at different cell temperatures in powder cells of : (a) ferrocenecarboxylic acid; (b) acetylferrocene; (c) ferrocenecarbaldehyde and (d) hydroxymethylferrocene.

The power-law dependence of photocurrent on time $(t)$ can be expressed as

$$
I_{p h} \propto t^{S^{\prime} p h} .
$$

The value of exponent $S_{p h}^{\prime}$ can be determined from the slope of the linear plot of log $I_{p h} v s$ log time obtained form the data taken at a fixed temperature. The slope $\left(S_{p h}^{\prime}\right)$ of 
the linear plots of $\log I_{p h}$ vs. log time for $\mathrm{FcH}$ derivatives have been found to be temperature dependent as presented in the Figures 3(a) to (d) for $\mathrm{Fc}(\mathrm{COOH}), \mathrm{Fc}\left(\mathrm{COCH}_{3}\right), \mathrm{Fc}(\mathrm{CHO})$ and $\mathrm{Fc}\left(\mathrm{CH}_{2} \mathrm{OH}\right)$, respectively. A drastic change in the plots of $S_{p h}^{\prime}$ vs $1 / T$ is evident in the studied temperature region. It could be mentioned here that a drastic change in the plots of $I_{p h}$ vs. $1 / T$ was also noticed (reported elsewhere) in the same temperature range.
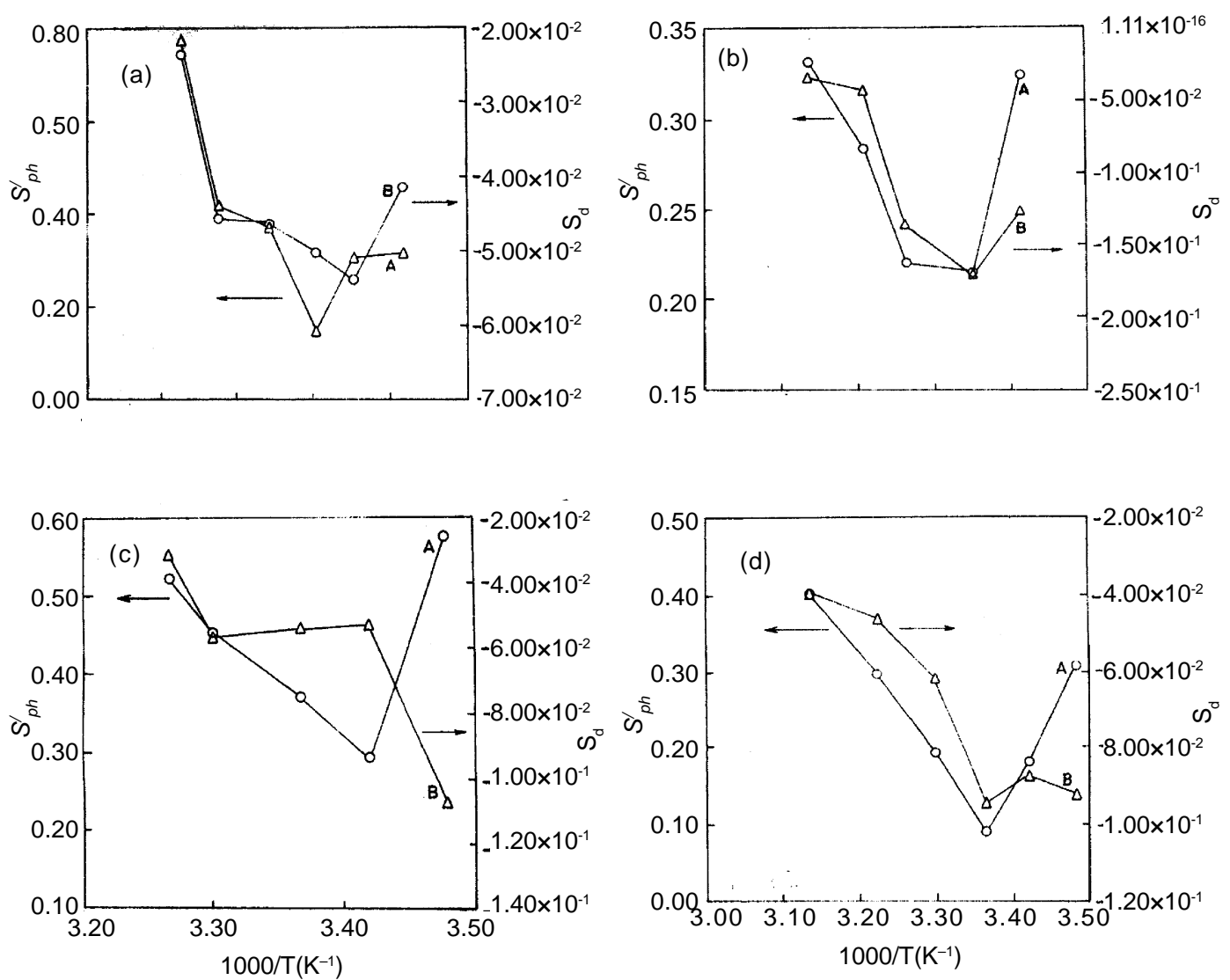

Figure 3. Linear plots of slopes $S^{\prime}{ }_{p h}$ and $S_{d} v s .1 / T$ (curve A corresponds to $S^{\prime}{ }_{p h}$, left scale; curve $B$ corresponds to $S_{d}$, right scale) in powder cells of : (a) ferrocenecarboxylic acid; (b) acetylferrocene; (c) ferrocenecarbaldehyde and (d) hydroxymethylferrocene.

Similar to the case of $I_{p h}$ in case of the $\mathrm{FcH}$ derivatives as stated above, the logarithm of current value $I_{d}$ for ferrocene derivatives under study after switching off light source did not show any linear relationship with time which indicates that the change in $I_{d}$ does not obey the first order kinetics $[18,19]$. In the present work the order of the kinetics of change in $I_{d}$ seems also to be complicated in nature.

For all the $\mathrm{FcH}$ derivatives studied the logarithmic plots of $I_{d}$ (after switching off light) has been observed to be linear with logarithm of time i.e., a power-law dependence of $I_{d}$ (before reaching the value nearly the original dark current) on time was noticed. The power-law dependence of $I_{d}$ on time can be expressed as 


$$
I_{d} \propto t^{S d}
$$

where, the value of exponent $S_{d}$ can be determined from the slope of the linear plot of log $I_{d} v s . \log t$. Such linear plots at different cell temperatures are presented in the Figures 4(a) to (d) for $\mathrm{Fc}(\mathrm{COOH}), \mathrm{Fc}\left(\mathrm{COCH}_{3}\right), \mathrm{Fc}(\mathrm{CHO})$ and $\mathrm{Fc}\left(\mathrm{CH}_{2} \mathrm{OH}\right)$, respectively. The slope $\left(S_{d}\right)$ of the linear plots depends on the cell temperature for these derivatives and the nature of the temperature dependence of $S_{d}$ is shown by the curve B's of the Figures 3(a) to (d). In these ferrocene derivatives, a drastic change in the nature of the temperature dependence of $S_{d}$ has been observed around certain temperature range. The experiment thus has demonstrated interestingly the temperature-dependent behavior of the kinetics of change in currents during illumination and after switching off light in case of the materials under study.
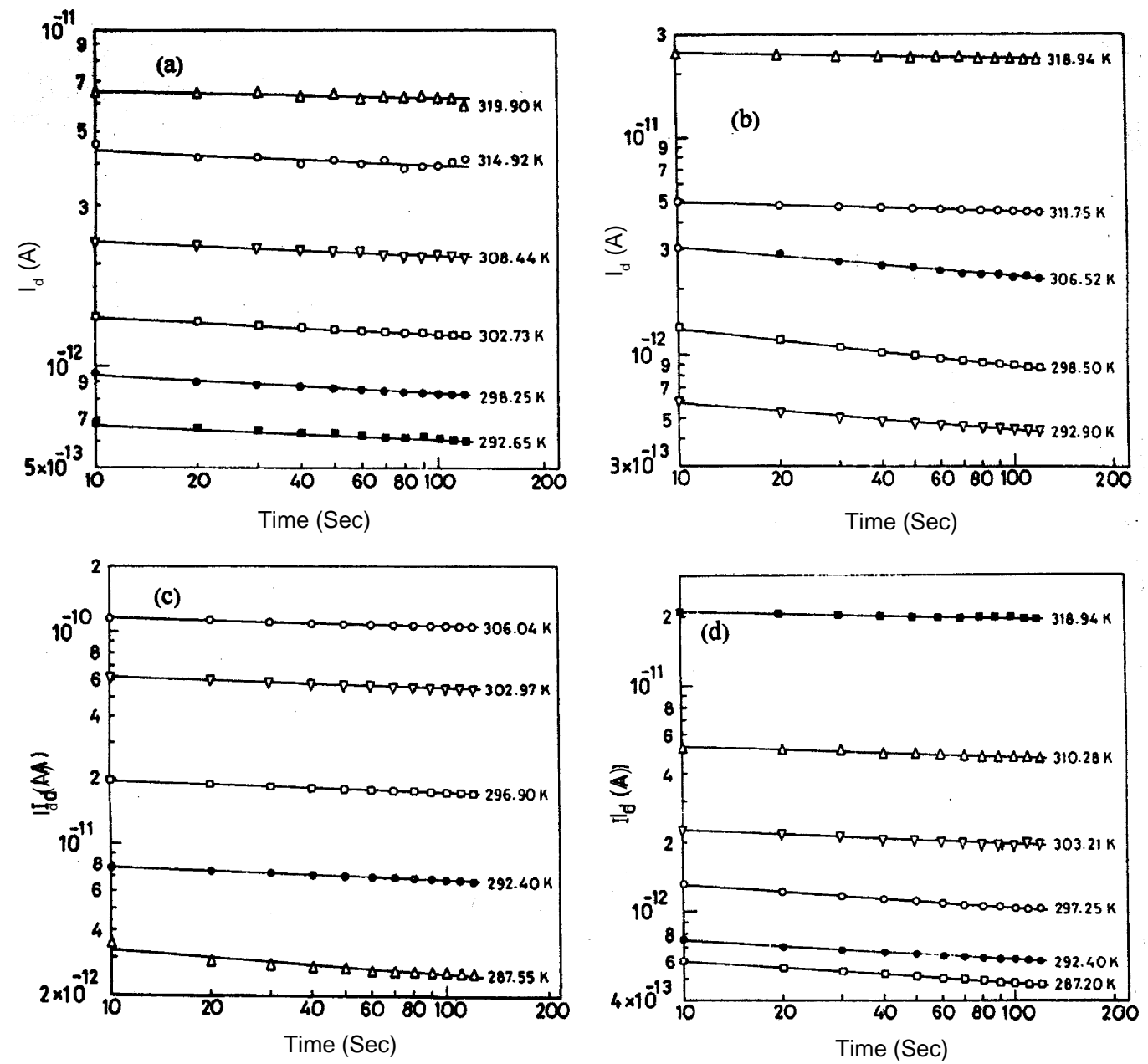

Figure 4. Plots of logarithm of $l_{d}$ values (after turning off light source) vs. logarithm of time at different cell temperatures in powder cell of : (a) ferrocenecarboxylic acid; (b) acetylferrocene; (c) ferrocenecarbaldehyde and (d) hydroxymethylferrocene. 
In some quasi-one dimensional molecular systems, photoinduced reversible phase transition has been reported $[22,23]$. The phenomenon has been characterized by evolution of the local molecular domains induced by photoexcitation. In some cases, the accumulated domains are observed to evolve into permanent and macroscopic phase conversion, that is the photoinduced phase transition. It has been concluded that some cooperative interaction is necessary for the individual local photoexcited species to evolve into the macroscopic phase conversion [24].

Phase transition occurs in $\mathrm{FcH}$ under the influence of external parameters such as temperature [25], pressure [26], adsorption of vapours [27] etc. Some FcH derivatives exhibit various kinds of mesophase transitions associated with intermolecular interactions [28]. From these observations it appears that occurrence of a phase transition, in $\mathrm{FcH}$ and its derivatives under suitable light illumination at a particular temperature is also possible. Fluctuations/ instabilities in physical parameters near phase transition region have been well recognized in various systems [29, 30]. In the present experiment, fluctuations/ instabilities in current (dark) have been observed after switching off the light source in all the ferrocene derivatives in a specific temperature range depending on the ferrocene derivative. Such an observation adds credence to the idea of photoinduced phase transition in the ferrocene derivatives under study. However, from the present study any direct evidence of the photoinduced phase transition in the materials under study could not be provided. The experimental results could be useful in the photoinduced redox reactions in relation to solar energy conversion and storage [31].

\section{Conclusions}

The temperature dependent unusual behavior of the kinetics of the change in currents during illumination and after switching off light in the solid state of some ferrocene derivatives under study possibly indicates that charge carrier trapping/ detrapping and recombination processes are significantly temperature dependent. A drastic change was noticed in $S_{p h}^{\prime}$ and $S_{d}$ around certain temperature range where time dependent anomalous changes in $I_{p h}$. during illumination of light were observed in the respective material. Such observation adds credence to the idea that the charge carrier trapping/detrapping and recombination processes have an important role in causing the temperature dependent anomalous photoconduction behavior of the ferrocene derivatives under study. Photoinduced reversible phase transition in these ferrocene derivatives is expected to be the cause of the unusual behavior of the kinetics of the change in currents duirng illumination and after switching off light source.

\section{Acknowledgments}

AKC thanks the Ministry of Human Resource Development (Department of Higher Education), Govt. of India, New Delhi, for allowing him to undertake research work at IACS, the authority of IACS, Kolkata-700 032, for providing a Research Associateship, laboratory and all other facilities, and also the Govt. of Bangladesh for granting him leave. 


\section{References}

[1] G L Geoffroy and M S Wrighton Organometallic Photochemistry (New York: Academic Press) p242 (1979)

[2] A F P Turner, I Karube and G S Wilson (eds) Biosensors (London : Oxford University Press) (1987)

[3] H Meier Organic Semiconductors (D-694 Weinheim : Verlag Chemie, GmbH) p165 (1974)

[4] R Ravanagh and J K Thomas Phys. Chem. Chem. Phys. 4193 (2002)

[5] M Even, B Heinrich, D Guillon, D M Guldi, M Prato and R Deschenaux Chem. Eur. J. 72595 (2001)

[6] R Frantz, F Carrie, J-O Durand and G F Lanneau New J. Chem. 25188 (2001)

[7] T Ma, K Inoue, K Yao, H Noma, T Shuji, E Abe, J Yu, X Wang and B Zhang J. Electroanal. Chem. 53731 (2002)

[8] X H Zhang, W Y Lai, T C Wong, Z Q Gao, Y C Jiang, S K Wu, H L Kwong, C G Lee and S T Lee Synth. Met. 114115 (2000)

[9] R N Bera and B Mallik Synth. Metals 9961 (1999)

[10] B Mallik and A. K. Chakraborty Phys. Stat. Sol. (a) 163395 (1997)

[11] R N Bera and B Mallik Solid State Commun. 108695 (1998)

[12] A Thander and B Mallik Chem. Phys. Letts. 330521 (2000)

[13] A K Chakraborty and B Mallik Synth. Met. 73239 (1995)

[14] H Meier Organic Semiconductors (D-694 Weinheim : Verlag Chemie, GmbH) p.310 (1974)

[15] B Mallik and A Bhattacharjee J. Phys. Chem. Solids 501113 (1989)

[16] K C Kao and W Hwang Electrical Transport in Solids (New York : Pergamon Press) p37 (1981)

[17] H Meier Organic Semiconductors (D-694 Weinheim : Verlag Chemie, GmbH) p68 (1974)

[18] J W Moore and R G Pearson Kinetics and Mechanism, (3rd ed.) (New York, Chichester, Brisbane, Toronoto : John Wiley and Sons) (1981)

[19] J H Espenson Chemical Kinetics and Reaction Mechanisms (2nd ed.) (New York, Singapore, New Delhi, Tokyo, Toronto etc. : McGraw-Hill, Inc.) (1995)

[20] E Ravasz, A L Somera, D A Mongru, Z N Oltvai and A-L Barabási Science 2971551 (2002)

[21] Albert-László Barabási and Réka Albert Science 286509 (1999)

[22] S Koshihara, Y Segawa, Y Tokura, T Koda and K Takeda Synth. Met. 55103 (1993)

[23] Y Tokura and S Koshihara Mol. Cryst. Liq. Cryst. 2163 (1992)

[24] S Koshihara J. Phys. Conf. Series 217 (2005)

[25] G Calverin, G Clec'n, J F Berar and D Andre J. Phys. Chem. Solids, 43785 (1982)

[26] B Karvally, B Mallik and G Kemeny J. Mater. Sci. Lett. 4912 (1985)

[27] A Bhattacharjee and B Mallik Bull. Chem. Soc. Jpn. 653462 (1992)

[28] K Iwai, M Katada, I Motoyama and H Sano Bull. Chem. Soc. Jpn. 601961 (1987)

[29] J Als-Nielsen in C Domb and M S Green (eds.) Phase Transitions and Critical Phenomena Vol. 5A (London, New York : Academic Press) p54 143 and 145 (1976)

[30] A G Naumovets Contemp. Phys. 30187 (1989)

[31] F Gutmann, H Keyzer and L E Lyons Organic Semiconductors (Malabar, Florida : Robert E. Krieger Pub. Co. Inc.) p360 and 463 (1983) 